\title{
Evaluation of different MRI parameters in benign prostatic hyperplasia-induced bladder outlet obstruction
}

\author{
Diaa Bakry Eldib ${ }^{1 *}$, Ayman Salah Moussa ${ }^{2}$ and Ahmed Sebaey ${ }^{3}$
}

\begin{abstract}
Background: Benign prostatic hyperplasia (BPH) is a common health problem faced by aging men and can be associated with bladder outlet obstruction (BOO). BPH patients usually suffer from lower urinary tract symptoms (LUTS) including obstructive and irritative urinary symptoms. The purpose of the current study was to clarify the correlation between MRI-derived prostate measurements and BPH-induced symptoms and findings including International Prostate Symptom Score (IPSS), uroflowmetry, and Postvoid residual urine (PVR).
\end{abstract}

Methods: In all, 34 patients (mean age was $58 \pm 8$ years, range 43 to 73 years) were examined by prostate MRI, uroflowmetry, postvoid residual urine, and fulfilled International Prostate Symptom Score questionnaire in this prospective study. The MRI-derived measurements including total prostate volume, transition zone volume, transition zone index, prostatic urethral angle, intravesical prostatic protrusion, and anterior fibromuscular stroma distance were correlated with International Prostate Symptom Score, uroflowmetry including maximum flow rate $\left(Q_{\max }\right)$ and $Q_{\max }$ grades, and postvoid residual urine using Pearson correlation coefficients $(r)$ and multiple linear regression. Relationship between BPH types was correlated with the same parameters using one-way analysis of variance.

Results: We found a strong significant correlation between total prostate volume, transition zone volume, and transition zone index with International Prostate Symptom Score, $Q_{\max }, Q_{\max }$ grades, and postvoid residual urine; however, in multiple linear regression analysis, all of them were found to be significant parameters for International Prostate Symptom Score. Also, our study showed a strong significant correlation between anterior fibromuscular stroma distance and intravesical prostatic protrusion with International Prostate Symptom Score and postvoid residual urine; however, in multiple linear regression analysis, only intravesical prostatic protrusion was found to be a significant parameter for postvoid residual urine. Our results also revealed a significant correlation between $\mathrm{BPH}$ types with $Q_{\max } Q_{\max }$ grades, International Prostate Symptom Score, and postvoid residual urine.

Conclusion: Several MRI-derived prostate parameters (total prostatic volume, transition zone volume, transition zone index, prostatic urethral angle, intravesical prostatic protrusion, anterior fibromuscular stroma distance, and BPH types) correlating significantly with the International Prostate Symptom Score, uroflowmetry parameters $\left(Q_{\max }\right.$ and $Q_{\max }$ grades), and postvoid residual urine.

Keywords: Benign prostatic hyperplasia, MRI, International Prostate Symptom Score, Uroflowmetry, Postvoid residual urine

\footnotetext{
* Correspondence: diaaeldeeb74@yahoo.com

${ }^{1}$ Radio-diagnosis Department, Faculty of Medicine, Benha University, Benha,

Egypt

Full list of author information is available at the end of the article
} 


\section{Background}

Benign prostatic hyperplasia (BPH) is a common health problem faced by aging men and can be associated with troublesome lower urinary tract symptoms (LUTS). Symptoms of BPH start to develop after the age of 40 years, and the incidence becomes greater than $50 \%$ by the age of 60 years and $90 \%$ by the age of 85 years [1]. Patients with BPH usually suffer from LUTS including obstructive urinary symptoms (incomplete emptying, intermittent voiding, straining, and weak stream) and irritative urinary symptoms (frequent voiding, nocturia, and urgency) [2].

Digital rectal examination (DRE), the International Prostate Symptom Score (IPSS), uroflowmetry, postvoid residual urine (PVR), serum prostate-specific antigen (PSA) levels, and imaging modalities, e.g., transrectal ultrasound (TRUS) are commonly used in the evaluation of $\mathrm{BPH}$ and also for follow-up of treatment outcome [2, 3].

TRUS is the primary modality in the evaluation of patients with $\mathrm{BPH}$ because it is feasible and widely available modality. However, there are some limitations of TRUS, e.g., being operator dependent, also its lower accuracy for volume measurement of the prostate compared with MRI $[3,4]$.

MRI is a well-known imaging modality in the diagnosis and follow-up of patients with prostate cancer, but in the assessment of BPH patients, the role MRI has been limited [5-7]. MRI offers high soft-tissue contrast resolution with delineation of prostatic lobar anatomy that allows BPH to be classified into different phenotypes [8]. MRI also provides precise measurements of the zonal and total volume of the prostate $[4,9]$.

International Prostate Symptom Score (IPSS), a wellknown clinical questionnaire, was developed by the WHO and commonly used for diagnosis and classification of the severity of LUTS. The IPSS questionnaire consists of seven items, each of which ranging from 0 (not at all) to 5 (almost all the time). The total score is the sum of the seven questions ranging from 0 to 35 . The patients are classified according to IPSS score into three groups: mild, 0-7; moderate, 8-19; and severe, $20-35[1,2]$.

Uroflowmetry is a non-invasive widely accessible inexpensive screening tool, used in the evaluation of men with $\mathrm{BPH}$, where urethral obstruction is very common [10]. Maximum flow rate $\left(Q_{\max }\right)$ is the most valuable parameter for prediction of bladder outlet obstruction (BOO). In case of $Q_{\max }<10 \mathrm{ml} / \mathrm{s}$, the likelihood of $\mathrm{BOO}$ is $90 \%$, while if $Q_{\max }$ ranging $10-15 \mathrm{ml} / \mathrm{s} 67 \%$ and in $Q_{\max }>15 \mathrm{ml} / \mathrm{s}$ only $30 \%$ [11]. The diagnostic accuracy of uroflowmetry for detecting BOO varies considerably. Therefore, it is limited as a diagnostic test as it is unable to discriminate between the underlying mechanisms [10].
Measurement of postvoid residual urine volume (PVR) is another noninvasive screening test for evaluating voiding dysfunction. Most urologists agree that volumes of 50-100 $\mathrm{ml}$ establish the lower threshold defining abnormal residual urine volume. Very large PVRs $(>300 \mathrm{ml})$ may be associated with an increased risk of hydronephrosis and renal function impairment [12].

MRI might be expensive in comparison to other imaging and urodynamic modalities, but the supreme softtissue contrast resolution and accurate data that MRI can provide lead to better treatment options for patients with $\mathrm{BPH}$-induced BOO. The aim of this study was to study the relationship between MRI-based prostate measurements and BPH-induced symptoms and findings parameters including International Prostate Symptom Score (IPSS), uroflowmetry, and postvoid residual urine (PVR).

\section{Methods}

We prospectively studied Middle Eastern men with LUTS due to BPH; MRI prostate studies, medical records, Uroflowmetry and fulfilled IPSS questionnaire data from 2017 to 2018. In all, 34 patients were recruited in the study. Informed written consent was signed by all subjects to share in the study after the explanation of its purposes. The study was approved by the Institutional Review Board of our hospital.

We excluded patients aged below 40 years, the presence of an indwelling urinary catheter, previous prostate surgery or pelvic radiation, urethral stricture, prostate or bladder cancer, neurogenic bladder disease, and patients on medical treatment for $\mathrm{BPH}$.

\section{MRI protocol}

MRI was performed using a $1.5 \mathrm{~T}$ body MRI system (Magnetom Essenza, Siemens, and Erlangen, Germany) and a pelvic phased-array coil. The applied MRI protocol was revised to assure of its competence with the study requirements.

MRI protocol consisted of non-contrast-enhanced T1weighted sequence obtained in the axial plane with repetition time/echo time [TR/TE]; 430/12 ms, section thickness; $3 \mathrm{~mm}$; interslice gap; $0.75 \mathrm{~mm}$, matrix size; $320 \times$ 216, field of view [FOV] of $280 \times 227 \mathrm{~mm}$, and scan time; $4 \mathrm{~min} 0 \mathrm{~s}$. T2-weighted sequence was also obtained in the axial, coronal, and sagittal planes with repetition time/echo time [TR/TE]; 3800/95 ms, section thickness; $3 \mathrm{~mm}$, interslice gap; $0.75 \mathrm{~mm}$, matrix size; $448 \times 336$, field of view [FOV]; $280 \times 320 \mathrm{~mm}$ and scan time; $3 \mathrm{~min}$ $0 \mathrm{~s}$. Fat-suppressed non-contrast-enhanced T1-weighted sequence [T1/FS] obtained in axial plane with repetition time/echo time [TR/TE] 434/13 ms; section thickness 3 $\mathrm{mm}$; interslice gap $0.75 \mathrm{~mm}$; matrix size $384 \times 288$; field of view [FOV] $350 \times 262 \mathrm{~mm}$; and scan time $4 \mathrm{~min}$ and $45 \mathrm{~s}$. STIR-weighted sequence was also obtained in the axial, coronal, and sagittal planes with repetition time/ 
echo time [TR/TE] 3310/39 ms; section thickness $3 \mathrm{~mm}$; interslice gap $0.75 \mathrm{~mm}$; matrix size $320 \times 240$; field of view [FOV] of $280 \times 227 \mathrm{~mm}$ and scan time $3 \mathrm{~min}$ and 7 s. Dynamic contrast-enhanced fat-suppressed T1weighted sequence $[\mathrm{T} 1 / \mathrm{FS}+\mathrm{C}]$ obtained in axial plane with repetition time/echo time [TR/TE] 4.49/2.29 ms; section thickness $3 \mathrm{~mm}$; interslice gap $0.3 \mathrm{~mm}$; matrix size $320 \times 240$; field of view [FOV] of $350 \times 307 \mathrm{~mm}$; and scan time 0 min and $16 \mathrm{~s}$. DW MRI was obtained in the axial plane with repetition time/echo time [TR/TE] 6000/92 ms; section thickness $4 \mathrm{~mm}$; interslice gap 1 $\mathrm{mm}$; matrix size $192 \times 126$; field of view [FOV] of $350 \times$ $280 \mathrm{~mm} ; b$ values of 50,400 , and $800 \mathrm{~s} / \mathrm{mm}^{2}$; and scan time $4 \mathrm{~min}$ and $49 \mathrm{~s}$. Apparent diffusion coefficient (ADC) maps obtained in axial DW-MRI sequences $\left(b\right.$ values $\left.=50-400-800 \mathrm{~s} / \mathrm{mm}^{2}\right)$. MRI images were reviewed and appropriate measurements were done by the radiologist author with more than 15 years of experience in MRI imaging.

\section{Image analysis and calculations}

T2-weighted images (WI) in axial, sagittal, and coronal planes were reviewed on Syngo ${ }^{\circ}$ MR D14 workstation.
$\mathrm{T}_{2}$-weighted images were used in our study, as the prostatic parenchyma and zonal anatomy were best evaluated on these images.

Prostate volume estimation was done using the Ellipsoid Prostate Volume Formula. The ellipsoid model is the original and de facto standard method of calculating the prostate volume. The greatest three dimensions of the prostate on axial and coronal $\mathrm{T}_{2-}$ weighted MRI images were measured manually and these measurements were used to determine the volume of the prostate using the ellipsoid formula: (Total prostate volume $(\mathrm{TPV})=$ anteroposterior $\times$ right-to-left $\times$ superoinferior $\times 0.52$ ). The entire prostate and the transition zone measurements obtained to calculate the total prostate volume (TPV), transition zone volume (TZV), and transition zone (TZ) index which was calculated by dividing the TZV by TPV (Fig. 1) [13, 14].

Prostatic urethral angle (PUA) was measured on midsagittal $\mathrm{T}_{2}$-weighted MR images, PUA was identified as the acute angle between the proximal and distal prostatic urethra (Fig. 2), similar to the methods published previously $[15,16]$.
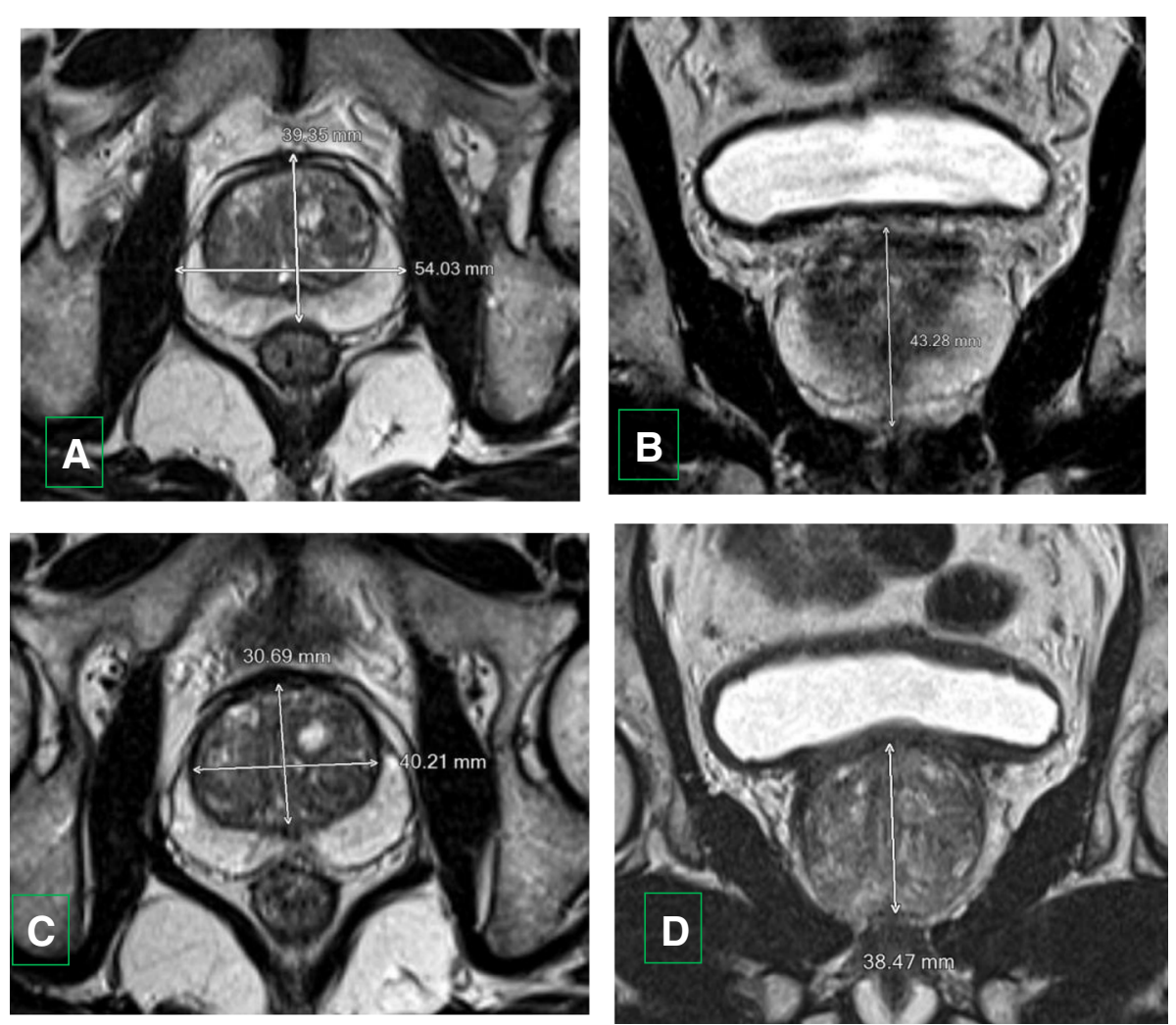

Fig. 1 Ellipsoid prostate volume measurement on axial and coronalT2-weighted MR images.a, b Total prostate volume (TPV) measurement. a Axial T2 WI showing anteroposterior and right-to-left dimensions. b Coronal T2 WI showing superoinferior dimension of the entire prostate. c, d Transition zone (TZ) volume (TZV) measurement. c Axial T2 WI showing anteroposterior and right-to-left dimensions. d Coronal T2 WI showing superoinferior dimension of the transition zone (TZ) of the prostate 

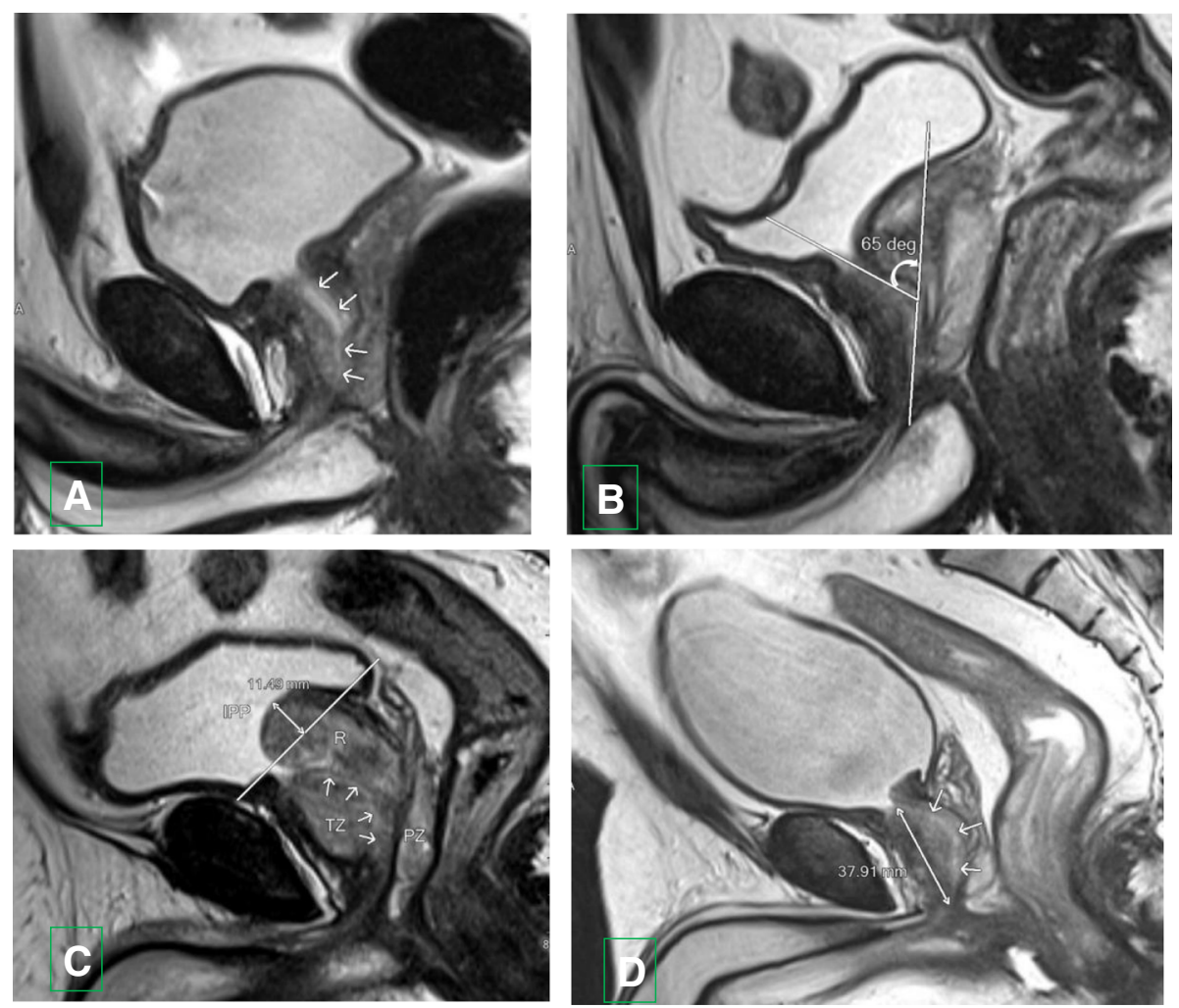

Fig. 2 Midsagittal T2-weighted MR images in different patients with BPH showing a The prostatic urethra (arrows). b Measurement of the prostatic urethral angle (PUA) (curved arrow); the acute angle between the proximal prostatic urethra and the distal prostatic urethra. c Measurement of the intravesical prostatic protrusion (IPP) (double-headed arrow). Retrourethral (median) lobe enlargement (R), transition zone (TZ), peripheral zone (PZ), and prostatic urethra (arrows) are noted. d Measurement of the AFMS distance (double-headed arrow). Prostatic urethra (arrows) is noted

Intravesical prostatic protrusion (IPP) was measured on the midsagittal $\mathrm{T}_{2}$-weighted MR image, IPP was identified as the distance from the tip of the protruding prostatic tissue perpendicular to the bladder circumference at the prostate base (Fig. 2), similar to the methods described in a previous study [17]. The degree of IPP was classified as grade 0 , no IPP; grade I, $\leq 5 \mathrm{~mm}$; grade II, > 5-10 $\mathrm{mm}$; and grade III, > $10 \mathrm{~mm} \mathrm{[17,18].}$

The anterior fibromuscular stroma (AFMS) is a muscular tissue that covers the entire ventral aspect of the prostate and extends from the bladder neck as a continuation of the detrusor muscle, covers up to the prostate apex, and consists of smooth muscle. The AFMS distance was measured on midsagittal $\mathrm{T}_{2}$-weighted MR image as supero-inferior distance extending from internal urethral orifice to the apex of the prostate along the ventral aspect of the prostate (Fig. 2) [19].

$\mathrm{BPH}$ types were identified according to the MRI classification described by Wasserman et al. [8], BPH types were identified on axial, sagittal, and coronal MR images. $\mathrm{BPH}$ phenotypes (Fig. 3) are the following: type $0 \mathrm{BPH}$; prostate volume $\leq 25 \mathrm{~cm}^{3}$ with no or little zonal enlargements, $\mathrm{BPH}$ type 1 ; bilateral transition zone (TZ) (lateral lobe) enlargement, BPH type 2; retrourethral (median) lobe enlargement, BPH type 3; bilateral transition zone (TZ) and retrourethral enlargement, BPH type 4; solitary or multiple pedunculated enlargement, $\mathrm{BPH}$ type 5; pedunculated with bilateral transition zone (TZ) and/or retrourethral enlargement, $\mathrm{BPH}$ type 6 ; subtrigonal or ectopic enlargement, and $\mathrm{BPH}$ type 7; additional combinations of zonal enlargements $[8,20]$.

\section{Urological and laboratory parameters}

IPSS questions were documented by patients in the outpatient clinic. PVR was measured by transabdominal ultrasound following uroflowmetry and registered. Prostatespecific antigen (PSA) results were also recorded including total PSA, free PSA, and PSA free/total ratio. All patients underwent urine analysis and culture to exclude any possible urinary tract infection.

Uroflowmetry results including maximum flow rate $\left(Q_{\max }\right)$, voided volume, flow time, and voiding time, as well as the shape of the curve, were registered. Uroflowmetry parameters were evaluated with voided volume > $150 \mathrm{ml}$ according to the EAU guidelines [10]. If the voided volume is less than $150 \mathrm{ml}$, the test was repeated the next day to confirm voided volume $\geq 150 \mathrm{ml}$. We classified our patients into three groups according to 


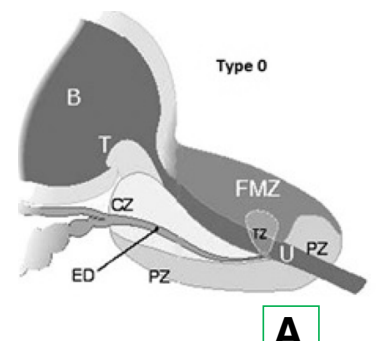

A

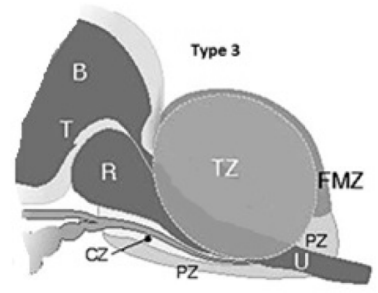

D

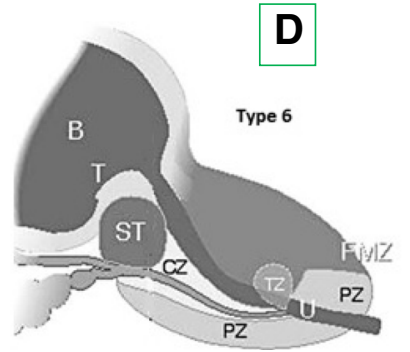

G
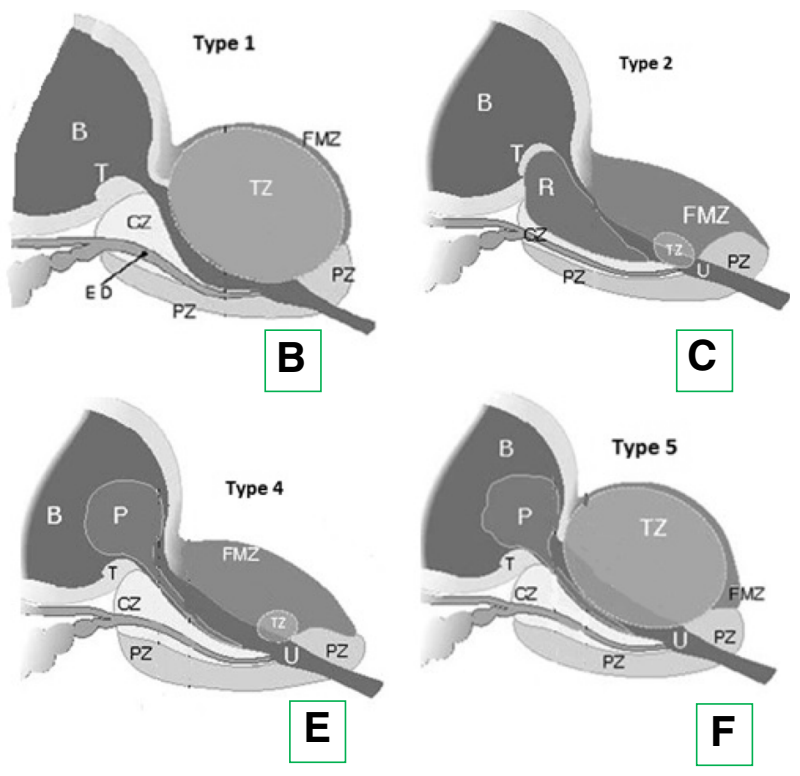

Fig. $3 \mathrm{BPH}$ types are as follows: a type $0 \mathrm{BPH}$; normal prostate volume with no or little zonal enlargements. b Type 1 BPH; transition zone (TZ) (lateral lobe) enlargement. c Type 2 BPH; retrourethral (median) lobe enlargement (R). d Type 3 BPH; transition zone (TZ) and retrourethral zone (R) enlargement. e Type $4 \mathrm{BPH}$; pedunculated adenoma. f Type $5 \mathrm{BPH}$; pedunculated adenoma with transition zone (TZ) enlargement. $\mathbf{g}$ Type 6 $\mathrm{BPH}$; subtrigonal enlargement. B, bladder; ED, ejaculatory duct; FMZ, fibromuscular zone; R, retrourethral (median) lobe; T, trigone; $\mathrm{U}$, urethra; $\mathrm{PZ}$, peripheral zone; TZ, transitional zone; CZ, central zone; P, pedunculated adenoma; ST, subtrigonal hyperplasia

maximum flow rate $\left(Q_{\max }\right)$ to generate three $Q_{\max }$ grades: unobstructed flow $\left(Q_{\max }>15 \mathrm{ml} / \mathrm{s}\right)$, equivocal flow $\left(Q_{\max } 10-15 \mathrm{ml} / \mathrm{s}\right)$, and obstructed flow $\left(Q_{\max }<10\right.$ $\mathrm{ml} / \mathrm{s})[12]$.

\section{Statistics}

All descriptive data are shown as means and standard deviation (SD), median, or numbers (\%). Pearson correlation coefficient $(r)$ was measured between $Q_{\max }, Q_{\max }$ grades, IPSS, PVR, and the sex continuous MRI-derived measurements; prostatic urethral angle (PUA), total prostatic volume (TPV), transition zone volume (TZV), transition zone (TZ) index (TZI), intravesical prostatic protrusion (IPP), and the anterior fibromuscular stroma (AFMS) distance.

Multiple linear regression analysis was calculated to identify the correlations between $Q_{\max }, Q_{\max }$ grades, IPSS, PVR, and MRI parameters.

A one-way analysis of variance (ANOVA) calculated to identify the correlations between MRI-based $\mathrm{BPH}$ types and $Q_{\max }, Q_{\max }$ grades, IPSS, and PVR.
Statistical significance was considered when $p$ value was less than 0.05. The statistical analysis was done using the SPSS program version 25.0 (Chicago, IL, USA).

\section{Results}

The patients' mean age was $58 \pm 8$ years, (range from 43 to 73 ) and median age 58 years. Their mean PUA was $57.5^{\circ} \pm 9.62^{\circ}$ (range, $45^{\circ}$ to $81.4^{\circ}$ ), and median was $54.25^{\circ}$. The mean total prostate volume (TPV) was $46.8 \pm 18.13 \mathrm{ml}$ (range, 27 to $97.47 \mathrm{ml}$ ), and median was $42 \mathrm{ml}$. The mean transition zone volume (TZV) was $34.1 \pm 10.3 \mathrm{ml}$ (range, 21 to $62.97 \mathrm{ml}$ ), and median was $32 \mathrm{ml}$. The mean transition zone index (TZI) was $0.75 \pm 0.12$ (range, 0.45 to 0.9 ), and median was 0.79 . The mean anterior fibromuscular stroma (AFMS) distance was $30.8 \pm 7 \mathrm{~mm}$ (range, 19.7 to $49.5 \mathrm{~mm}$ ), and median was $30.6 \mathrm{~mm}$. The mean intra-vesical prostatic protrusion (IPP) was 3.3 $\pm 6.47 \mathrm{~mm}$ (range, 0 to $29.3 \mathrm{~mm}$ ), and median was 0 mm. (Table 1). 
Table 1 Mean $\pm S D$, range and median values of age, PUA, TPV, TZV, TZI, AFMS distance, IPP, $Q_{\text {max }}$ IPSS, and total PSA

\begin{tabular}{llll}
\hline & Mean \pm SD & Range & Median \\
\hline Age (years) & $58 \pm 8$ & $43-73$ & 58 \\
PUA $\left(^{\circ}\right)$ & $57.5 \pm 9.62$ & $45-81.4$ & 54.25 \\
TPV $(\mathrm{ml})$ & $46.8 \pm 18.13$ & $27-97.47$ & 42 \\
TZV $(\mathrm{ml})$ & $34.1 \pm 10.3$ & $21-62.97$ & 32 \\
TZI & $0.75 \pm 0.12$ & $0.45-0.9$ & 0.79 \\
AFMS distance $(\mathrm{mm})$ & $30.8 \pm 7$ & $19.7-49.5$ & 30.6 \\
IPP $(\mathrm{mm})$ & $3.3 \pm 6.47$ & $0-29.3$ & 0 \\
$Q_{\text {max }}(\mathrm{ml} / \mathrm{s})$ & $18 \pm 8.24$ & $3.7-32$ & 15.25 \\
IPSS score & $12.7 \pm 7.76$ & $5-30$ & 9.5 \\
PVR (ml) & $69.8 \pm 63$ & $5-240$ & 53 \\
Total PSA (ng/ml) & $3.1 \pm 2.74$ & $0.34-8.94$ & 2.1
\end{tabular}

PUA prostatic urethral angle; TPV total prostate volume; TZV transition zone volume; TZI transition zone index; IPP intravesical prostatic protrusion; AFMS anterior fibromuscular stroma; $Q_{\max }$ maximum flow rate; IPSS International Prostate Symptom Score; PVR postvoid residual urine; PSA prostatic specific antigen

The mean maximum flow rate $\left(Q_{\max }\right)$ was $18 \pm$ $8.24 \mathrm{ml} / \mathrm{s}$ (range, 3.7 to $32 \mathrm{ml} / \mathrm{s}$ ), and median was $15.25 \mathrm{ml} / \mathrm{s}$. The mean International Prostate Symptom Score (IPSS) score was $12.7 \pm 7.76$ (range, 5 to 30 ) and median was 9.5. The mean postvoid residual urine (PVR) was $69.8 \pm 63 \mathrm{ml}$ (range, 5 to $240 \mathrm{ml}$ ), and median was $53 \mathrm{ml}$. The mean total PSA was 3.1 $\pm 2.74 \mathrm{ng} / \mathrm{ml}$ (range, 0.34 to $8.94 \mathrm{ng} / \mathrm{ml}$ ), and median was $2.1 \mathrm{ng} / \mathrm{ml}$. (Table 1 ).

$Q_{\max }$ grades were normal flow in 17 patients $(50 \%)$, borderline flow in 12 patients (35.3\%), and obstructed flow in 5 patients $(14.7 \%)$. IPP grades were grade 0 in 23 patients $(67.6 \%)$, grade 1 in 4 patients $(11.8 \%)$, grade 2 in 3 patients $(8.8 \%)$, and grade 3 in 4 patients $(11.8 \%)$.

The maximum flow rate $\left(Q_{\max }\right)$ was significantly correlated with the MRI-derived measurements including TPV, TZV, TZI, and IPP (Table 2). TPV $(p=0.013)$, TZV $(p=0.016)$, TZI $(p=0.016)$, and IPP $(p=0.014)$.
There was a significant correlation of $Q_{\max }$ grades with the following MRI-derived measurements: TPV, TZV, TZI, and IPP (Table 2). TPV $(p=0.001), \operatorname{TZV}(p \leq$ $0.001)$, TZI $(p=0.023)$, and IPP $(p=0.009)$.

Notably, there was a significant correlation of IPSS with the following MRI-derived measurements: TPV, TZV, TZI, IPP, and AFMS distance (Table 2). TPV ( $p \leq$ $0.001), \operatorname{TZV}(p \leq 0.001)$, TZI $(p=0.015), \operatorname{IPP}(p \leq$ $0.001)$, and AFMS distance $(p=0.027)$.

The postvoid residual urine (PVR) was significantly correlated with the following MRI-derived measurements: PUA, TPV, TZV, TZI, IPP, and AFMS distance (Table 2). PUA $(p=0.032), \operatorname{TPV}(p \leq 0.001), \operatorname{TZV}(p=$ $0.001)$, TZI $(p=0.011)$, IPP $(p \leq 0.001)$, and AFMS distance $(p=0.026)$.

There was no significant correlation between the maximum flow rate $\left(Q_{\max }\right), Q_{\max }$ grades, and IPSS with PUA. Also, there was no significant correlation between the maximum flow rate $\left(Q_{\max }\right)$ and $Q_{\max }$ grades with AFMS distance (Table 2).

Parameters for multiple linear regression of maximum flow rate $\left(Q_{\max }\right), Q_{\max }$ grades, IPSS, and postvoid residual urine (PVR) are presented in (Table 3). Only TPV, TZV, and TZI were found to be predictors of IPSS ( $p=0.015$, 0.005 , and 0.011 respectively). TZV and TZI were found to be predictors of $Q_{\max }$ grades ( $p=0.02$ and 0.037 respectively. IPP was the only predictors of PVR $(p=0.023)$.

BPH types on MRI were as follows: type $0(n=0)$, type $1(n=18)$ (Fig. 4$)$, type $2(n=0)$, type $3(n=13)$ (Fig. 5$)$, type $4(n=0)$, type $5(n=3)$ (Figs. 6 and 7$)$, type $6(n=$ $0)$, and type $7(n=0)$. Our results revealed a significant correlation between MRI-based $\mathrm{BPH}$ types with $Q_{\max }$ $(p=0.016), Q_{\max }$ grades $(p=0.033)$, IPSS $(p=$ $0.013)$, and $\operatorname{PVR}(p=0.001)$, in one-way analysis of variance (ANOVA) analysis.

\section{Discussion}

$\mathrm{BPH}$ develops as non-malignant growth of the prostate gland, very commonly observed in aging men. BPH

Table 2 Pearson correlation coefficients ( $r$ ) between $Q_{\max }, Q_{\max }$ grades, IPSS, PVR and PUA, TPV, TZV, TZI, IPP, AFMS distance

\begin{tabular}{llllllll}
\hline & & PUA & TPV & TZV & TZI & IPP & AFMS distance \\
\hline$Q_{\max }$ & $r$ & -0.094 & -0.420 & -0.408 & 0.446 & -0.415 & -0.286 \\
& $p$ & 0.5964 & $0.0133^{*}$ & $0.0167^{*}$ & $0.01605^{*}$ & $0.0147^{*}$ & 0.1010 \\
$Q_{\max }$ grades & $r$ & 0.157 & 0.523 & 0.554 & -0.511 & 0.437 & 0.289 \\
& $p$ & 0.3750 & $0.0015^{*}$ & $0.0007^{*}$ & $0.02317^{*}$ & $0.0098^{*}$ & 0.0971 \\
IPSS & $r$ & 0.209 & 0.590 & 0.617 & -0.551 & 0.543 & 0.379 \\
& $p$ & 0.2360 & $0.0002^{*}$ & $0.0001^{*}$ & $0.01513^{*}$ & $0.0009^{*}$ & $0.0272^{*}$ \\
PVR & $r$ & 0.367 & 0.569 & 0.531 & -0.576 & 0.694 & 0.381 \\
& $p$ & $0.0328^{*}$ & $0.0004^{*}$ & $0.0012^{*}$ & $0.01146^{*}$ & $<0.0001^{*}$ & $0.0261^{*}$ \\
\hline
\end{tabular}

PUA prostatic urethral angle; TPV total prostate volume; TZV transition zone volume; TZI transition zone index; IPP intravesical prostatic protrusion; AFMS anterior fibromuscular stroma; $Q_{\max }$ maximum flow rate; $P V R$ postvoid residual urine; IPSS International Prostate Symptom Score Statistical significance ( $p$ values with asterisk) was considered at the critical level of 0.05 
Table 3 Parameters for multiple linear regression between $Q_{\max } Q_{\max }$ grades, IPSS, PVR and age, PUA, TPV, TZV, TZI, IPP, AFMS distance

\begin{tabular}{|c|c|c|c|c|c|c|c|c|}
\hline & & Age & PUA & TPV & TZV & TZI & IPP & AFMS distance \\
\hline$Q_{\max }$ & $p$ & 0.783 & 0.533 & 0.218 & 0.176 & 0.206 & 0.482 & 0.823 \\
\hline$Q_{\max }$ grades & $p$ & 0.372 & 0.835 & 0.058 & $0.020^{*}$ & $0.037^{*}$ & 0.235 & 0.562 \\
\hline IPSS & $p$ & 0.215 & 0.776 & $0.015^{*}$ & $0.005^{*}$ & $0.011^{*}$ & 0.060 & 0.944 \\
\hline PVR & $p$ & 0.484 & 0.384 & 0.579 & 0.453 & 0.637 & $0.023^{*}$ & 0.539 \\
\hline
\end{tabular}

PUA prostatic urethral angle; TPV total prostate volume; TZV transition zone volume; TZI transition zone index; IPP intravesical prostatic protrusion; AFMS anterior fibromuscular stroma; $Q_{\max }$ maximum flow rate; PVR postvoid residual urine; IPSS International Prostate Symptom Score

Statistical significance ( $p$ values with asterisk) was considered at the critical level of 0.05

occurs as an age-related phenomenon in nearly all men, starting to develop after the age of 40 years. BPH is troublesome to patients when it is combined with lower urinary tract symptoms (LUTS) [21].

MRI is a commonly used imaging modality for diagnosis and follow-up of patients with prostate cancer, but using MRI in the assessment of BPH patients still has limited value. This research has been conducted to investigate the role of MRI in assessing $\mathrm{BPH}$ and related symptoms. Statistical analysis and results demonstrated that different MRI measurements of the prostate including PUA, TPV, TZV, TZ index, IPP, and AFMS distance showed significant correlation with maximum flow rate $\left(Q_{\max }\right), Q_{\max }$ grades, IPSS, and PVR.

In our study, we found a strong significant correlation between TZV, TZI, TPV, and IPSS to $Q_{\max }, Q_{\max }$ grades, and PVR; however, in multiple linear regression analysis, all of them were found to be a significant parameter for IPSS. Also, our study showed a strong correlation between AFMS distance and IPP with IPSS and PVR; however, in a multiple linear regression model, only IPP was identified to be a significant parameter for PVR.

The relationship between TZV and TPV as regards the IPSS was controversial. Guneyli et al. [2] described a significant correlation between TZV and TPV with IPSS. However, Kaplan et al. [14] described a stronger correlation between TZV and IPSS and a weak correlation between TPV and IPSS. Kaplan et al. [14] also found a significant correlation between TZI and IPSS. A TZI $\geq 0.5$ was correlated with IPSS. Notably, Kurita et al. [22] measured TZI on TRUS and demonstrated that TZI was a significant predictor for IPSS, and TZI was a predictor of acute urinary retention (AUR) in BPH patients when TZI $\geq 0.65$. However, there are few studies reported no relationship between either the TZV or TZI and IPSS measured on TRUS $[1,23]$. Our data confirmed a significant correlation between TZV, TPV, TZI, and IPSS.

We acknowledge that some of the dilemmas in the literature may rise from the inaccurate prostate volume measured on TRUS. TRUS is an operator-dependent imaging modality, together with the irregular contours of an enlarged prostate, limit the accuracy of prostate volume measurements [24]. MRI proved to be a more reliable method in determining prostate volumes than TRUS [13].

Intravesical prostatic protrusion (IPP) is a protrusion of the prostate into the bladder as a result of prostatic enlargement. IPP is a valuable predictor for evaluating BOO. IPP leads to ball-valve type of BOO and inhibition of the funnel effect of the bladder neck at urination [25, 26]. Chia et al. [18] stated that powerful bladder contraction opens the canal between the lateral prostate lobes, but exaggerates the ball and valve effect of IPP, which induces more BOO.

The relationship between the $\mathrm{BPH}$-related findings and IPP was stated before based on TRUS findings
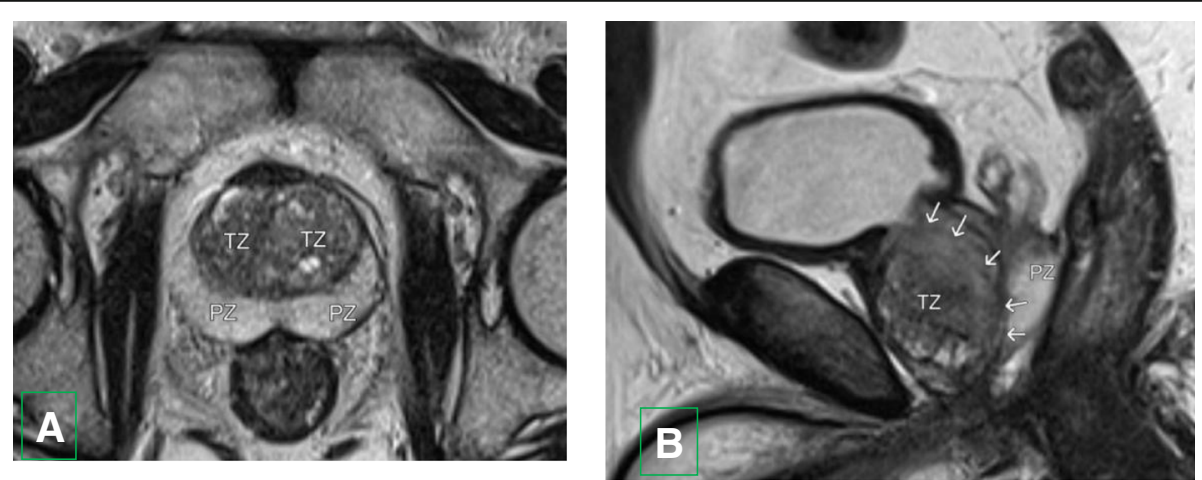

Fig. 4 Sixty-two years old man with type $1 \mathrm{BPH}$. a Axial T2 WI showing bilateral transition zone (TZ) enlargement. Peripheral zone (PZ) is noted. b Sagittal T2 WI showing transition zone (TZ) enlargement displacing the prostatic urethra posteriorly (arrows). No retrourethral enlargement. Peripheral zone (PZ) is noted 

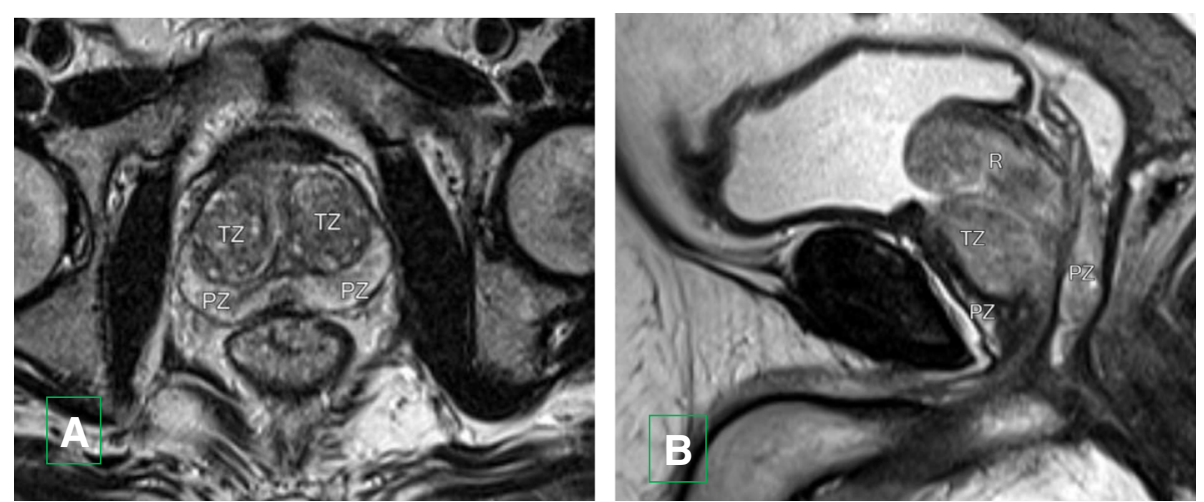

Fig. 5 Sixty-nine years old man with type $3 \mathrm{BPH}$. a Axial T2 WI showing bilateral transition zone (TZ) (lateral lobe) enlargement. Peripheral zone (PZ) is noted. b Sagittal T2 WI showing transition zone (TZ) and retrourethral (median) lobe enlargement (R). Peripheral zone (PZ) is noted

$[1,27]$. Similar to these TRUS studies, our study demonstrated a significant correlation of MRI-based IPP measurement with all of the parameters studied; $Q_{\max }, Q_{\max }$ grades, PVR, and IPSS. Notably, in multilinear regression analysis, IPP was an independent predictor for PVR only.

Nishio et al. [19] reported shortening of the AFMS distance before beginning of the voiding process. The percent contraction of the AFMS from before voiding to just before initiation of voiding was $11 \%$ in the normal voiding group and $1 \%$ in the voiding dysfunction group $(p<0.05)$. Hocaoglu et al. [28, 29] documented shortening of the AFMS distance during voiding process than that before voiding in their real-time MRI studies (rtMRI). In our study, we found that the AFMS distance was significantly longer in patients with higher PVR and IPSS.

Only a few studies reported the correlation between the IPSS and PUA. Park et al [1] found that higher PUA $\geq 34^{\circ}$ was significantly correlated with IPSS in BPH patients. In contrast to this study, Guneyli et al. [2] stated that PUA had no significant correlation with IPSS, which is in line with our findings.

In our study, PUA was highly significant and correlated with PVR, but PUA had no significant correlation with $Q_{\max }$ and $Q_{\max }$ grades. In contrast to our results, Cho et al. [15], using the fluid dynamic model and mathematical simulations, reported that PUA is inversely correlated with maximum flow rate $\left(Q_{\max }\right)$. This controversy could be attributed to the criteria of patients in Cho et al. [15] study. In the study by Cho et al. [15], patients were healthy men (aged 50-59 years, prostate volume $30 \mathrm{ml}$, and $Q_{\max } 15 \mathrm{ml} / \mathrm{s}$ ) with no evidence of BOO.

Our results revealed a significant correlation between MRI-based BPH types with $Q_{\max }, Q_{\max }$ grades, IPSS, and PVR. In contrast to our study, Guneyli et al. [2] reported that there was no correlation between MRI-based BPH types and IPSS.

Our study has several limitations. It is a study with a relatively small sample size. The final histologypathology results for all of the patients in our study were
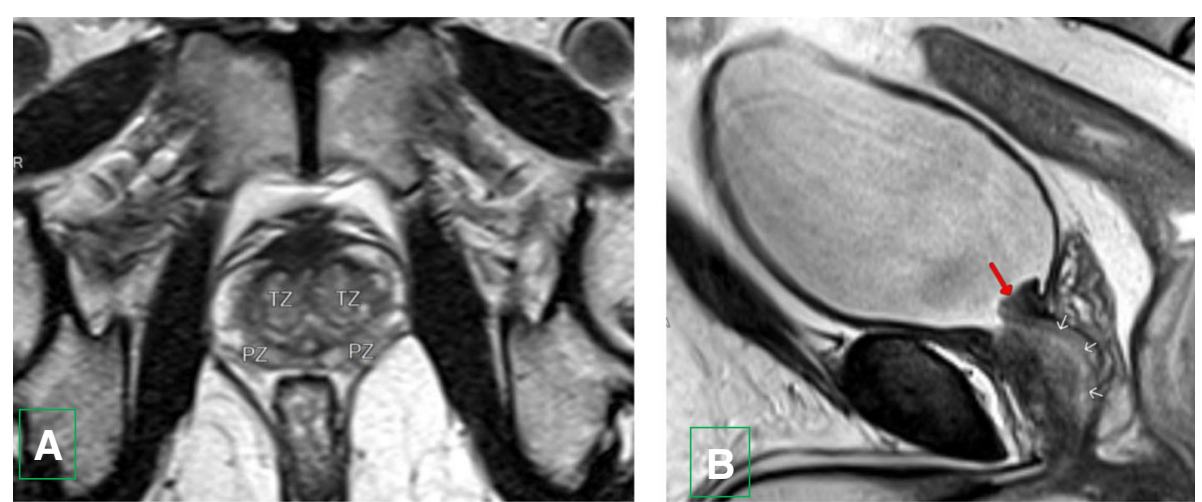

Fig. 6 Sixty-three years old man type $5 \mathrm{BPH}$. a Axial T2 WI showing bilateral transition zone (TZ) (lateral lobe) enlargement. Peripheral zone (PZ) is noted. b Sagittal T2 WI showing pedunculated enlargement (red arrow) protruding into the bladder base. No retrourethral (median) lobe enlargement along the posterior aspect of the prostatic urethra (arrows) 

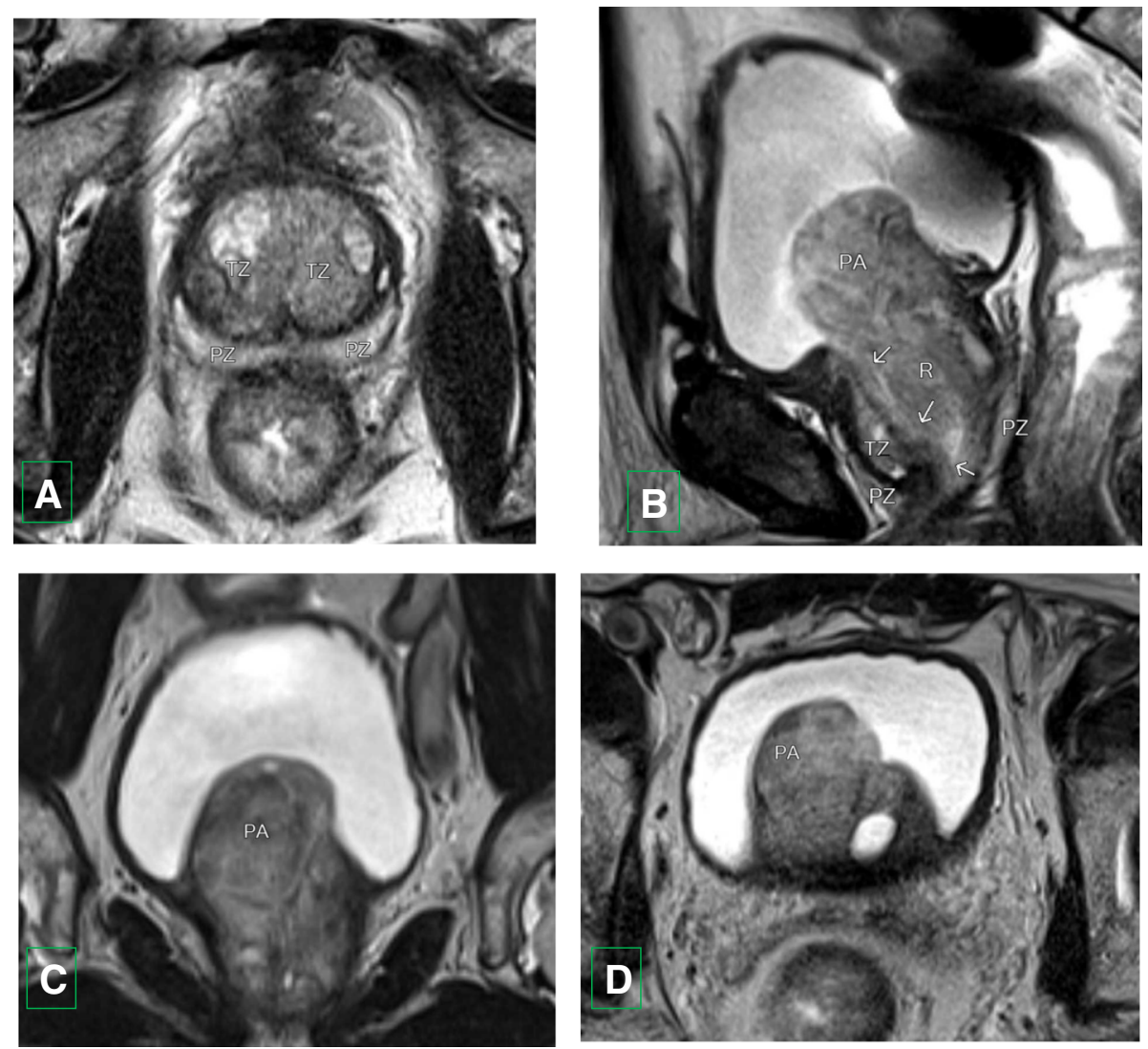

Fig. 7 Seventy-three years old man type $5 \mathrm{BPH}$. a Axial T2 WI showing bilateral transition zone (TZ) (lateral lobe) enlargement. Peripheral zone (PZ) is noted. b Sagittal T2 WI showing pedunculated adenoma (PA) protruding into the bladder base. Retrourethral (median) lobe enlargement (R) along the posterior aspect of the prostatic urethra (arrows). c Coronal and $\mathbf{d}$ axial T2 WI showing pedunculated adenoma (PA) protruding into the bladder base

not available. However, to our knowledge, this is the first study among Middle Eastern men and a unique study that investigates MRI-derived prostatic measurements with uroflowmetry and PVR, as two wellknown parameters accepted as diagnostic tools for $\mathrm{BPH}$ and $\mathrm{BOO}$ evaluation.

\section{Conclusion}

We identified several MRI-based prostate measurements (TPV, TZV, TZI, IPP, PUA, and AFMS distance) correlating significantly with the IPSS, $Q_{\max }, Q_{\max }$ grades, and PVR. MRI is a useful non-invasive imaging modality for describing the anatomy and clinical parameters of the prostate in patients with BPH. MRI can lead to better comprehension and enhanced treatment choice of patients with $\mathrm{BPH}$.

\section{Abbreviations}

AFMS: Anterior fibromuscular stroma; BOO: Bladder outlet obstruction; $\mathrm{BPH}$ : Benign prostatic hyperplasia; DRE: Digital rectal examination; EAU: European Association of Urology; IPP: Intravesical prostatic protrusion; IPSS: International Prostate Symptom Score; LUTS: Lower urinary tract symptoms; MRI: Magnetic resonance imaging; PSA: Prostatespecific antigen; PUA: Prostatic urethral angle; PVR: Postvoid residual urine; $Q_{\max }:$ Maximum flow rate; rtMRI: Real-time magnetic resonance imaging; TPV: Total prostate volume; TRUS: Transrectal ultrasound; TZI: Transition zone index; TZV: Transition zone volume

\section{Acknowledgements}

The authors thank all the study participants for their patience and support.

\section{Authors' contributions}

DBE suggested and developed the research idea, reviewed the literature, collected and analyzed the data, performed the statistical analysis, wrote and revised the manuscript, prepared the MRI cases, performed the required measurements, and prepared the figures and tables. ASM suggested and developed the research idea, collected and analyzed the data, reviewed the literature, shared in statistical analysis, and edited the manuscript. AS collected, analyzed, and interpreted the data, and revise the written manuscript. All authors read and approved the final manuscript.

\section{Funding}

This study had no funding from any resource.

\section{Availability of data and materials}

The datasets used and/or analyzed during the current study are available from the corresponding author on reasonable request.

\section{Ethics approval and consent to participate}

This study was approved by the Research Ethics Committee of the Faculty of Medicine at Benha University in Egypt. All patients included in this study gave written informed consent to participate in this research. If the patient was less than 16 years old or unconscious at the time of the study, written 
informed consent for their participation was given by their parent or legal guardian.

\section{Consent for publication}

All patients included in this research gave written informed consent to publish the data contained within this study. If the patient was less than 16 years old, deceased, or unconscious when consent for publication was requested, written informed consent for the publication of this data was given by their parent or legal guardian.

\section{Competing interests}

The authors declare that they have no competing interests.

\section{Author details}

${ }^{1}$ Radio-diagnosis Department, Faculty of Medicine, Benha University, Benha, Egypt. ${ }^{2}$ Urology Department, Faculty of Medicine, Beni Suef University, Beni Suef, Egypt. ${ }^{3}$ Urology Department, Faculty of Medicine, Benha University, Benha, Egypt.

Received: 7 June 2019 Accepted: 5 August 2019

Published online: 06 September 2019

\section{References}

1. Park YJ, Bae KH, Jin BS, Jung HJ, Park JS (2012) Is increased prostatic urethral angle related to lower urinary tract symptoms in males with benign prostatic hyperplasia/lower urinary tract symptoms? Korean J Urol 53(6): 410-413. https://doi.org/10.4111/kju.2012.53.6.410

2. Guneyli S, Ward E, Peng Y, Nehal Yousuf A, Trilisky I, Westin C, Oto A (2017) MRI evaluation of benign prostatic hyperplasia: Correlation with international prostate symptom score. J Magn Reson Imaging 45(3):917-925. https://doi. org/10.1002/jmri.25418

3. Grossfeld GD, Coakley FV (2000) Benign prostatic hyperplasia: clinical overview and value of diagnostic imaging. Radiol Clin North Am 38(1):31-47

4. Karademir I, Shen D, Peng $Y$ et al (2013) Prostate volumes derived from MR and volume-adjusted serum prostate-specific antigen: correlation with Gleason score of prostate cancer. AJR Am J Roentgenol 2013 201:1041-1048

5. Emad-Eldin S, Halim M, Metwally LIA, Abdel-Aziz RM (2014) Diffusionweighted MR imaging and ADC measurement in normal prostate, benign prostatic hyperplasia and prostate carcinoma. Egypt J Radiol Nucl Med 45(2):535-542. https://doi.org/10.1016/j.ejrnm.2014.02.017

6. Elian MMM, Abdel Gawad EA, Fathelbab TKH (2015) Value of functional MRI in evaluation of patients with suspected prostate cancer. Egypt J Radiol Nucl Med 46(4):1183-1191. https://doi.org/10.1016/j.ejrnm.2015.07.007

7. Ali TFT, ElHariri MA, Riad MM (2018) Diffusion-weighted MRI in prostatic lesions: Diagnostic performance of normalized ADC using normal peripheral prostatic zone as a reference. Egypt J Radiol Nucl Med 49(1):239-244. https://doi.org/10.1016/j.ejrnm.2017.09.007

8. Wasserman NF, Spilseth B, Golzarian J, Metzger GJ (2015) Use of MRI for lobar classification of benign prostatic hyperplasia: potential phenotypic biomarkers for research on treatment strategies. AJR Am J Roentgenol 205(3):564-571. https://doi.org/10.2214/AJR.14.13602

9. Turkbey, B., Fotin, S. V., Huang, R. J., Yin, Y., Daar, D., Aras, O., ... Choyke, P. L. (2013). Fully automated prostate segmentation on MRI: comparison with manual segmentation methods and specimen volumes. AJR Am J Roentgenol, 201(5), W720-W729. doi:https://doi.org/10.2214/AJR.12.9712

10. Gravas, S., Bach, T., Bachmann, A., Drake, M., Gacci, M., \& Gratzke, C. (2018): EAU guidelines on the management of non-neurogenic male lower urinary tract symptoms (LUTS), incl. benign prostatic obstruction (BPO). EAU Guidelines. Edn. presented at the EAU Annual Congress Copenhagen 2018. ISBN 978-94-92671-01-1. Available at: https://uroweb.org/wp-content/ uploads/EAU-Guidelines-on-the-Management-of-Non-neurogenic-MaleLUTS-2018-large-text.pdf

11. de la Rosette, J. J., G. Alivizatos, S. Madersbacher, M. Perachino, D. Thomas, F. Desgrandchamps, M. de Wildt and U. (2001): European Association of Urology "EAU Guidelines on benign prostatic hyperplasia (BPH)." Eur Urol 40(3): 256-263; discussion 264

12. Kelly CE (2004) Evaluation of voiding dysfunction and measurement of bladder volume. Rev Urol 6(Suppl 1):S32-S37

13. Garvey B, Turkbey B, Truong H, Bernardo M, Periaswamy S, Choyke PL (2014) Clinical value of prostate segmentation and volume determination on MRI in benign prostatic hyperplasia. Diagn Interv Radiol 20(3):229-233. https:// doi.org/10.5152/dir.2014.13322

14. Kaplan SA, Te AE, Pressler LB, Olsson CA (1995) Transition zone index as a method of assessing benign prostatic hyperplasia: correlation with symptoms, urine flow and detrusor pressure. J Urol 154(5):1764-1769

15. Cho KS, Kim JH, Kim DJ, Choi YD, Kim JH, Hong SJ (2008) (2008): Relationship between prostatic urethral angle and urinary flow rate: its implication in benign prostatic hyperplasia pathogenesis. Urology 71:858-862

16. Ku JH, Ko DW, Cho JY, Oh SJ (2010) Correlation between prostatic urethral angle and bladder outlet obstruction index in patients with lower urinary tract symptoms. Urology 75(6):1467-1471. https://doi.org/10.1016/j.urology.2 009.08.049

17. Nose H, Foo KT, Lim KB, Yokoyama T, Ozawa H, Kumon H (2005) Accuracy of two noninvasive methods of diagnosing bladder outlet obstruction using ultrasonography: intravesical prostatic protrusion and velocity-flow video urodynamics. Urology 65:493-497

18. Chia SJ, Heng CT, Chan SP et al (2003) Correlation of intravesical prostatic protrusion with bladder outlet obstruction. BJU Int. 2003 91:371-374

19. Nishio K, Soh S, Syukuya T et al (2014) Role of male pelvic floor muscles and anterior fibromuscular stroma in males on a (1)-blocker treatment: a magnetic resonance imaging study. Int J Urol 21:724-727

20. Wasserman NF (2006) Benign prostatic hyperplasia: a review and ultrasound classification. Radiol Clin 44(5):689-710

21. Roehrborn, C. G. (2005). "Benign prostatic hyperplasia: an overview." Rev Urol 7 Suppl 9: S3-S14. PMID: 16985902

22. Kurita Y, Masuda H, Terada H, Suzuki K, Fujita K (1998) Transition zone index as a risk factor for acute urinary retention in benign prostatic hyperplasia. Urology 1998 51:595-600

23. Lepor H, Nieder A, Feser J, O'Connell C, Dixon C (1997) Total prostate and transition zone volumes, and transition zone index are poorly correlated with objective measures of clinical benign prostatic hyperplasia. J Urol 1997 158:85-88

24. Choi YJ, Kim JK, Kim HJ, Cho KS (2009) Interobserver variability of transrectal ultrasound for prostate volume measurement according to volume and observer experience. AJR Am J Roentgenol 2009 192:444-449

25. Kuo HC (1999) Clinical prostate score for diagnosis of bladder outlet obstruction by prostate measurements and uroflowmetry. Urology 1999 54:90-96

26. Keqin Z, Zhishun X, Jing Z, Haixin W, Dongqing Z, Benkang S (2007) Clinical significance of intravesical prostatic protrusion in patients with benign prostatic enlargement. Urology 2007 70:1096-1099

27. Kim KH, Kim YS (2010) Correlation of male overactive bladder with intravesical prostatic protrusion. Korean J Urol 2010 51:843-846

28. Hocaoglu Y, Roosen A, Herrmann K, Tritschler S, Stief C, Bauer RM (2012) Real-time magnetic resonance imaging (MRI): anatomical changes during physiological voiding in men. BJU Int 2012 109:234-239

29. Hocaoglu Y, Herrmann K, Walther $S$ et al (2013) Contraction of the anterior prostate is required for the initiation of micturition. BJU Int 2013 111:1117-1123

\section{Publisher's Note}

Springer Nature remains neutral with regard to jurisdictional claims in published maps and institutional affiliations.

\section{Submit your manuscript to a SpringerOpen ${ }^{\circ}$ journal and benefit from:}

- Convenient online submission

- Rigorous peer review

- Open access: articles freely available online

- High visibility within the field

- Retaining the copyright to your article

Submit your next manuscript at $>$ springeropen.com 